\title{
Fabrication of Self-Standing Silver Nanoplate Arrays by Seed-Decorated Electrochemical Route and Their Structure-Induced Properties
}

\author{
Guangqiang Liu, 1 Guotao Duan, ${ }^{1}$ Lichao Jia, ${ }^{1}$ Jingjing Wang, ${ }^{1}$ Hongzhi Wang, \\ Weiping Cai, ${ }^{1}$ and Yue $\mathrm{Li}^{2}$ \\ ${ }^{1}$ Key Laboratory of Materials Physics and Anhui Key Laboratory of Nanomaterials and Nanotechnology, Institute of Solid State Physics, \\ Chinese Academy of Sciences, Hefei 230031, China \\ ${ }^{2}$ Center of Medical Physics and Technology, Hefei Institute of Physical Science, Chinese Academy of Sciences, Hefei 230031, China
}

Correspondence should be addressed to Guangqiang Liu; liugq@issp.ac.cn

Received 3 September 2012; Accepted 12 December 2012

Academic Editor: Bingqiang Cao

Copyright (c) 2013 Guangqiang Liu et al. This is an open access article distributed under the Creative Commons Attribution License, which permits unrestricted use, distribution, and reproduction in any medium, provided the original work is properly cited.

\begin{abstract}
We present an electrochemical route to synthesize silver nanoplates on seed-decorated Indium tin oxide (ITO) glass substrate. The nanoplates are several tens of to several hundred nanometers in dimension. The density of nanoplates covered on the substrate can be controlled well by adjusting the amounts of seed. All the nanoplates are standing on the substrate uniformly even at very high density. Silver nanoplate arrays displayed an extraordinary superhydrophobicity after chemical modification and can serve as highly active surface-enhanced Raman scattering (SERS) substrates for microdetection. The arrays can also be used as electrodes for electrochemical capacitor with high power density.
\end{abstract}

\section{Introduction}

Surface morphology and assembly behavior of nanostructured materials decide their performances; therefore, their investigations have attracted much interest in recent years originated from their widespread applications in optics, electronics, optoelectronics, information storage, self-cleaning, catalysis biological, chemical sensing, surface-enhanced Raman scattering (SERS) effect, and so forth [1-10]. Among these, SERS is a typical instance, which is mainly determined by the surface morphology and structure of noble metal materials [11]. It means a dramatic enhancement of intensity of the Raman spectra of the molecule absorbed on the surface of metal [12-17]. Some reports have revealed that sharp protrusion and nanogaps in highly roughed metallic surface, so called active "hot" spots, are responsible for SERS, because of larger electromagnetic enhancement caused by local surface plasma resonance at these sites $[18,19]$. In addition, other properties, that is, self-cleaning surface, use as electrode for electrochemical capacitor, are also determined by the surface morphology [20-28]. Self-cleaning surface should have static contact angles (CA) of water droplets larger than $150^{\circ}$ and the slid angle (SA) smaller than $10^{\circ}$, which can be prepared by a combination of reducing the surface energy and enhancing the surface roughness [2934]. The self-cleaning performance can be widely used for preventing the adhesion of water or snow on the surface of some objects. In electrochemical capacitor, electrochemical reaction occurred at the interface between electrode and electrolyte, so a large electrochemical interface of electrode is favorable to the enhancement of the power density [35]. In whole, assembly of nanostructured material with high rough surface is the key factor in the above applications.

Previously, we reported a spin-coated Ag seed growth method to fabricate standing silver nanoplate arrays with high rough surfaces [36]. But through this method, the obtained silver nanoplates are not sufficiently tightly anchored on indium tin oxide (ITO) substrate. Additionally, these nanoplates are not uniform in size and distribution when we need nanoplate array with a more high distribution density covered on the substrate for some applications. The main reason is poor uniform distribution of $\mathrm{Ag}$ seeds 


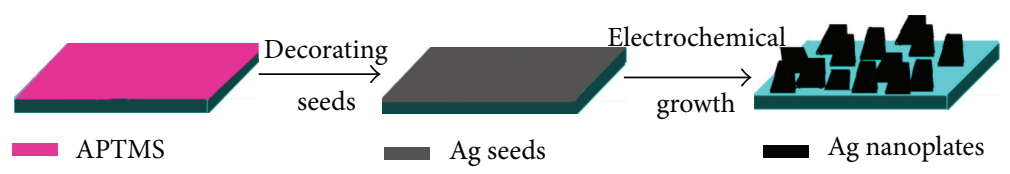

(a)

(b)

(c)

FIGURE 1: Schematic illustration of formation of nanoplate arrays: (a); a layer of 3-aminopropyltrimethoxysilane (APTMS) absorbed on clean ITO (b) Ag seeds decorated on clean ITO; (c) growth of Ag nanoplate arrays by electrochemical method.

with a high density by spin-coating method. In this paper, we report a simple electrochemical approach decorated by Ag seeds to synthesize silver nanoplates with controllable density (roughness) covered on the ITO substrate. The obtained silver nanoplates are covered on the substrate uniformly with very high density. The whole procedure was shown in Figure 1. Firstly, we decorated a layer of 3-aminopropyltrimethoxysilane (APTMS) on clean ITO (Figure 1(a)), and then this substrate was immersed into as-prepared Ag colloid solution for decorating Ag seeds on the substrate (Figure 1(b)), followed growth of Ag nanoplates by electrochemical deposition route on the substrate directly. The as-prepared silver nanoplate arrays demonstrated structured enhanced SERS activity and a good superhydrophobic property after chemical modification with $n$-dodecanethiol, which has important potential application in fluidic device, self-cleaning surfaces, organic molecule detection, and so forth. In addition, the arrays can also be used as electrode for electrochemical capacitor with high power density.

\section{Experiment}

2.1. Decorating Seeds. The details of preparing silver seeds have been reported previously [36]. Briefly, $60 \mathrm{mM} \mathrm{AgNO}_{3}$ of $0.5 \mathrm{~mL}$ and $35 \mathrm{mM}$ sodium citrate of $1 \mathrm{~mL}$ were added into $98 \mathrm{~mL}$ distilled water. The mixed solution was stirred for five minutes. Then, an aqueous $20 \mathrm{mM} \mathrm{NaBH}_{4}$ solution of $0.5 \mathrm{~mL}$, which had been aged at room temperature for $2 \mathrm{~h}$, was added quickly, stirred for $1 \mathrm{~h}$, and then aged at room temperature for $24 \mathrm{~h}$ before use. ITO substrates were ultrasonically cleaned in acetone and then in ethanol for $1 \mathrm{~h}$. Cleaned ITO substrates were placed into a dilute solution of 3-aminopropyltrimethoxysilane (APTMS) $(0.3 \mathrm{~mL}$ of APTMS in $3 \mathrm{~mL}$ of methanol) for 12 hours and rinsed with methanol to remove redundant APTMS. The polymer-coated substrate was immersed in above Ag seeds colloid solution for different time, $4 \mathrm{~h}, 8 \mathrm{~h}$, and $10 \mathrm{~h}$ respectively. Then, the substrates were taken out and dried with high-purity flowing nitrogen.

2.2. Electrochemical Deposition. Silver nitrate $\left(\mathrm{AgNO}_{3}\right.$, $99+\%)$, and poly(vinyl pyrrolidone) (PVP, MW = 30000 ) were purchased from Shanghai Chemical Reagent Co. All chemicals were analytically graded and were used without further purification. Typically, $0.05 \mathrm{~g} \mathrm{AgNO}_{3}$ and $0.25 \mathrm{~g}$ PVP were added to $50 \mathrm{~mL}$ of water followed by stirring till complete dissolution. Such aqueous solution was used as electrolyte for electrodeposition. The electrochemical deposition was carried out under current density $5 \mu \mathrm{A} / \mathrm{cm}^{2}$ at room temperature. A graphite flake was used as anode and the Ag seed-decorated ITO substrate as cathode. After deposition for about $5 \mathrm{~h}$, the substrate with products was taken out, cleaned with distilled water for several times and dried with high-purity flowing nitrogen for characterization and property study.

2.3. Characterization. The as-prepared samples were characterized by X-ray diffraction (XRD) (Philips X' pert-PRO, $\mathrm{Cu} \mathrm{K} \alpha(0.15418 \mathrm{~nm})$ radiation), field emission scanning electronic microscope (FESEM, sirion 200 FEG).

Before contact angle (CA) measurement, the substrate was immersed in ethanol solution of $n$-dodecanethiol $(1 \times$ $10^{-3} \mathrm{M}$ ) for about $10 \mathrm{~h}$ and washed in turn with ethanol and water and dried with nitrogen to coat a self-assembled monolayer of low free energy materials. Distilled water droplets with a volume of $5 \mu \mathrm{L}$ were used in contact angle test.

For Raman spectral measurement, the samples were immersed in 4-aminothiophenol (4-ATP) solution with different concentration for $30 \mathrm{~min}, 10^{-6} \mathrm{~mol} / \mathrm{L}, 10^{-8} \mathrm{~mol} / \mathrm{L}$, $10^{-10} \mathrm{~mol} / \mathrm{L}$, and $10^{-12} \mathrm{~mol} / \mathrm{L}$, respectively. After that, the substrates were taken out from the solution and rinsed with de-ionized water, and then dried with high-purity flowing nitrogen. The Raman spectra were recorded on a macroscopic confocal Raman spectrometer, using a laser beam with an excitation wavelength of $532 \mathrm{~nm}$.

For electrochemical measurements, a typical threeelectrode cell was employed. The electrolyte was $5 \mathrm{M} \mathrm{KOH}$ solution. An $\mathrm{Hg} / \mathrm{HgO}$ electrode and a platinum electrode were used as the reference electrode and the counter electrode.

\section{Results and Discussion}

After electrodeposition on the ITO substrate coated with the Ag-seeds, the samples were characterized by XRD firstly, as shown in Figure 2(A) (curve (a), (b), (c) corresponding to samples obtained preimmersed Ag seeds colloid solution for $4 \mathrm{~h}, 8 \mathrm{~h}, 10 \mathrm{~h}$ ), and the intensity of diffraction increase gradually with increasing immersing time. Four diffraction peaks $(2 \theta)$ at $38.2,44.3,64.4$, and $77.3^{\circ}$ were observed, corresponding to the (111), (200), (220), and (311) of the face-centered cubic $(f c c)$ structure of silver with space group Fm3m (JCPDS), respectively.

Figures 2(B)-2(D) display SEM image of as-prepared samples. We can see that these units are nanoplates with 

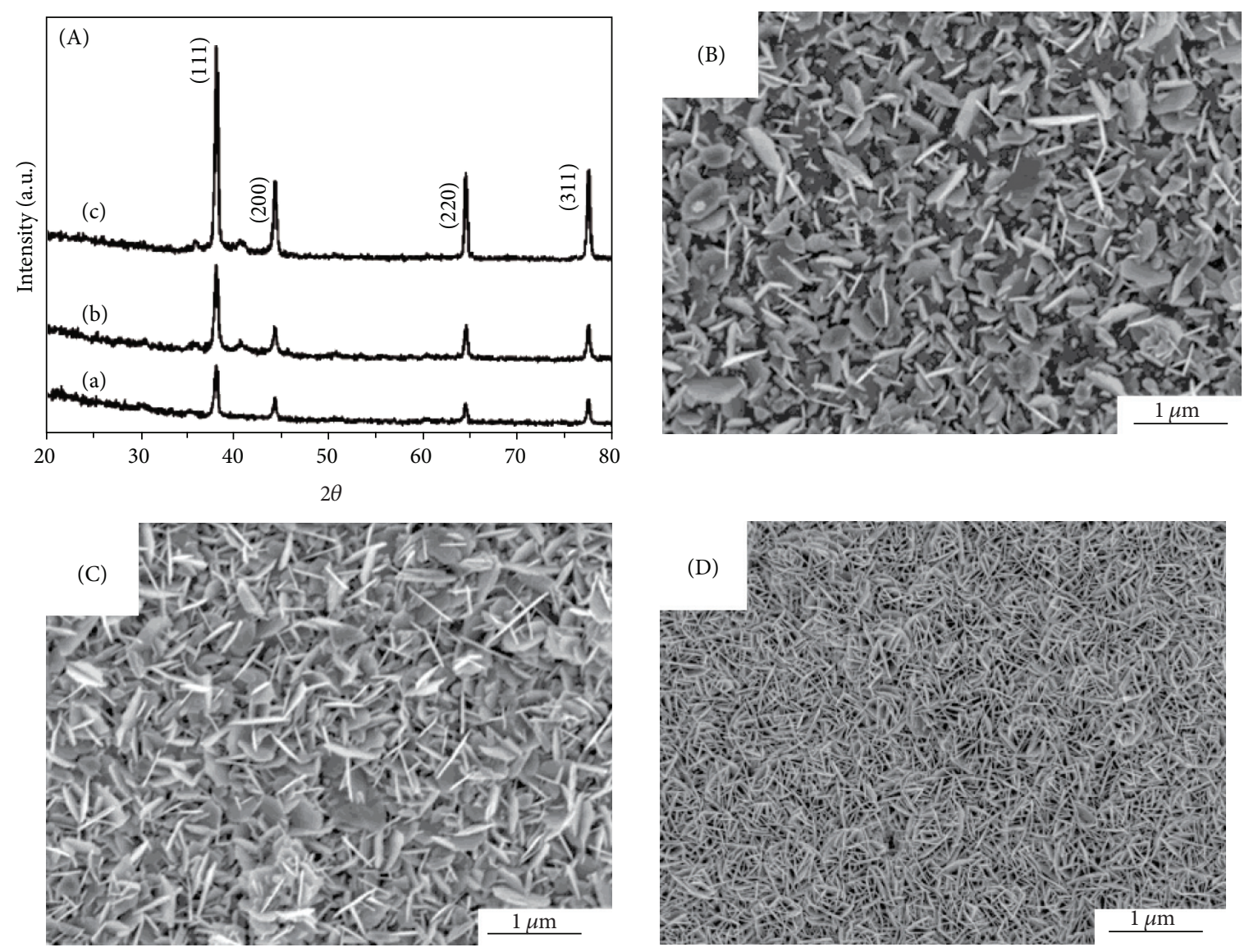

FIGURE 2: XRD of the as-prepared sample with the polymer-coated substrate immersing in Ag seeds colloid solution for different time, $4 \mathrm{~h}$ (curve a), $8 \mathrm{~h}$ (curve b), and $10 \mathrm{~h}$ (curve c), respectively; FESEM images of the samples prepared with the polymer-coated substrate immersing in $\mathrm{Ag}$ seeds colloid solution for different time, $4 \mathrm{~h}(\mathrm{~B}), 8 \mathrm{~h}(\mathrm{C}), 10 \mathrm{~h}(\mathrm{D})$.

the size in several tens to several hundred nanometers. Obviously, the densities of coverage of the nanoplates become dense with enlarging time of polymer-decorated ITO substrate into $\mathrm{Ag}$ colloid solution, and the density of coverage is about $7.2 \times 10^{7}$ plates $/ \mathrm{cm}^{2}, 4.0 \times 10^{8}$ plates $/ \mathrm{cm}^{2}$, and $3.5 \times 10^{9}$ plates $/ \mathrm{cm}^{2}$, respectively. When the nanoplates are very dense, all nanoplates are standing on the ITO substrate uniformly and cross-linking together with each other, leading to a very rough surface (see Figure 2(D)), while some nanoplates are gathered together at high density fabricated by our previous method. But when the nanoplates are sparse, most of them are lying on the ITO substrate, and hence the surface roughness is low (Figure 2(B)). It demonstrates that we can also control the density of coverage of Ag nanoplates through decorating Ag seed layer method. That is, the roughness of the surface can be tuned.

\section{The Application of Ag Nanoplate Arrays}

4.1. Superhydrophobicity. The water droplet shapes on the surface of three samples (Figures 2(B)-2(D)) with different density is shown in Figure 3. The water CAs were $132^{\circ}, 150^{\circ}$ and $160^{\circ}$ respectively, (Figures $3(\mathrm{a})-3(\mathrm{c})$ ) and the SA is about $7^{\circ}$ for nanoplates arrays with highest density (Figure 2(D)). It is obviously that surface roughness is directly related to the $\mathrm{CA}$. With increasing of density of Ag nanoplates, the surface roughness is increasing, and the CA turned lager accordingly. The spherical water droplet (Figure 3(d)) also can further confirm the superhydrophobicity. We describe the CA in terms of the Cassie equation [37]:

$$
\cos \theta_{r}=f_{1} \cos \theta-f_{2},
$$

where $\theta_{r}$ and $\theta$ are the contact angles of a rough (textured) surface and a flat surface, respectively, $f_{1}$ and $f_{2}$ are the fractional interfacial areas of the $\mathrm{Ag}$ nanoplates arrays and the air interspaces among the $\mathrm{Ag}$ nanoplates arrays $\left(f_{1}+\right.$ $\left.f_{2}=1\right)$. From this equation, it is easy to conclude that increasing the value of $f_{2}$ can increase CA. For a flat surface modified with the $n$-dodecanethiol, the CA is $108^{\circ}$, so $f_{2}$ is calculated to be 0.91 . This means that the larger fraction of air $\left(f_{2}\right)$ among the interspaces of standing Ag nanoplate arrays with higher density leads to superhydrophobicity of the surface. Another feature of a water-repellent surface from the dynamic viewpoint is a low sliding angle, which enables a water drop to easily roll down the surface. This means that these silver nanoplate arrays with a rough surface trap a large amount of air among nanoplate structures, leading to a very good superhydrophobicity with self-cleaning effect. 

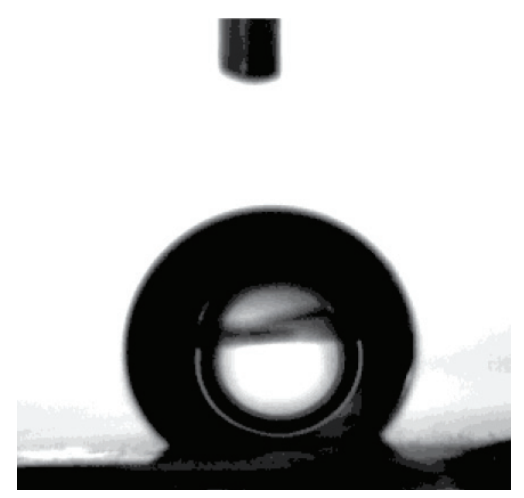

(a)

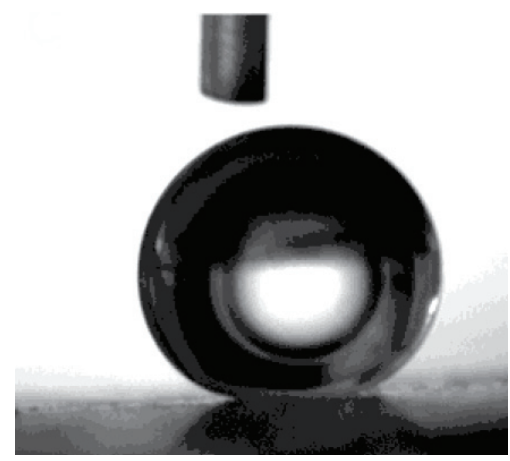

(c)

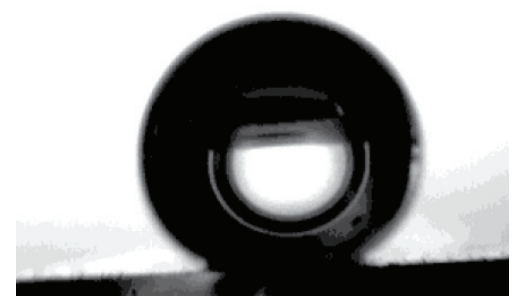

(b)

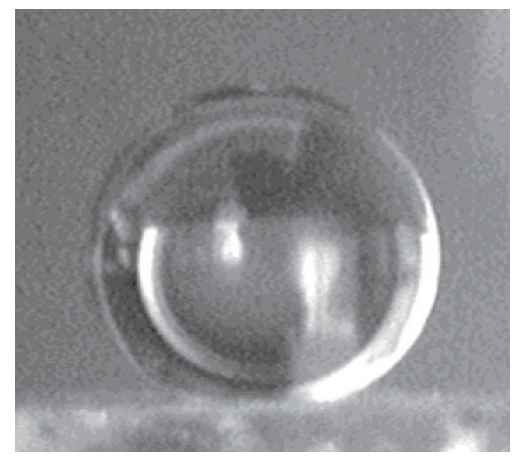

(d)

Figure 3: Photo images of water droplets on the surface of Ag nanoplates arrays with the polymer-coated substrate immersing in Ag seeds colloid solution for different time, $4 \mathrm{~h}(\mathrm{a}), 8 \mathrm{~h}(\mathrm{~b})$, and $10 \mathrm{~h}$ (c), respectively, and the water CAs were $132^{\circ}, 150^{\circ}$, and $160^{\circ}$; (d) photograph of (c).

4.2. Surface-Enhanced Raman Scattering Effect. We use these three samples with different density of Ag nanoplates covered as substrates to study the SERS activity, as shown in Figure 4 corresponding to the Raman spectra of 4-ATP molecular (after immersion in $10^{-6} \mathrm{~mol} / \mathrm{L}$ 4-ATP solution for $10 \mathrm{~min}$ ). We can find the SERS activity raises with increase of the distribution density of $\mathrm{Ag}$ nanoplates arrays (integrating time $1 \mathrm{~s}$ ). In order to further probe into the detection limit of SERS property of the substrate with highest density Ag nanoplate arrays (shown in Figure 2(D)), three different concentrations $\left(10^{-8} \mathrm{M}, 10^{-10} \mathrm{M}, 10^{-12} \mathrm{M}\right)$ of 4-ATP were examined. The Raman spectral intensity of 4-ATP turned weaker with decrease of the concentration of 4-ATP in a certain range (integrating time $1 \mathrm{~s}$ ). But, even when the concentration of 4-ATP was decreased to $10^{-12} \mathrm{M}$, we still can distinguish Raman peaks of 4-ATP (see curve (c) in Figure 5). This evidence indicated that this rough surface built with Ag nanoplates arrays had an excellent SERS performance. We attribute this enhancement to the many protrusions of standing $\mathrm{Ag}$ nanoplates and interstitials or nanogaps is formed among these nanoplates, which provide many hot "spots" for SERS $[38,39]$. Obviously, with increasing the density of Ag nanoplates, the "hot spots" increase correspondingly.

4.3. Electrochemical Properties of Silver Nanoplates Arrays. The cyclic voltammetry at different scan rate using silver nanoplates arrays with high density (Figure 2(D)) as electrode

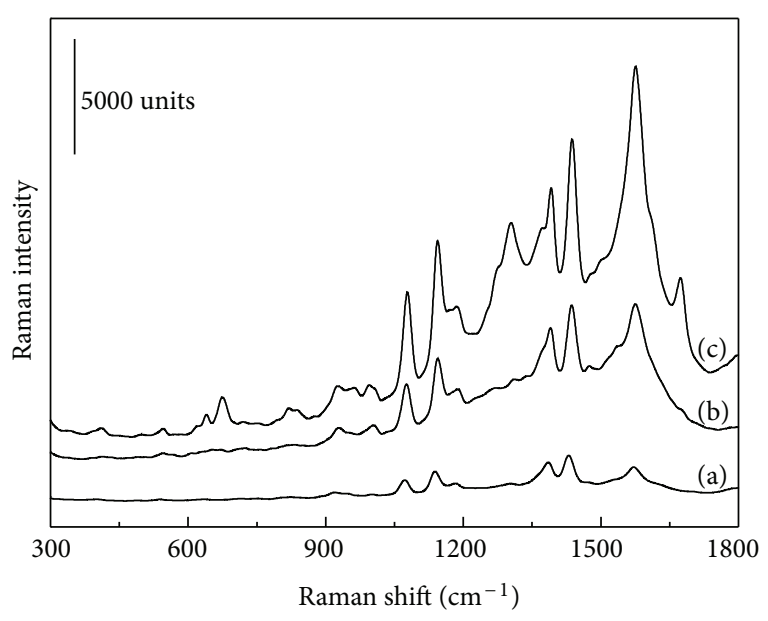

Figure 4: Raman spectra of the 4-ATP on different surface of Ag nanoplates arrays (Figure 2(D)) with the polymer-coated substrate immersing in Ag seeds colloid solution for different time, $4 \mathrm{~h}$ (a), $8 \mathrm{~h}$ (b), $10 \mathrm{~h}(\mathrm{c})$, respectively.

in $5 \mathrm{M} \mathrm{KOH}$ solution was shown in Figure 6. It can be noted that the shape of $\mathrm{CV}$ curves have no change and the redox peak is at the same position with different scan rates, which illustrate that the silver nanoplates electrodes has a good reversibility. Figure 7 shows the charge and discharge curve. After 25 cycles, the efficiency of charge/discharge 


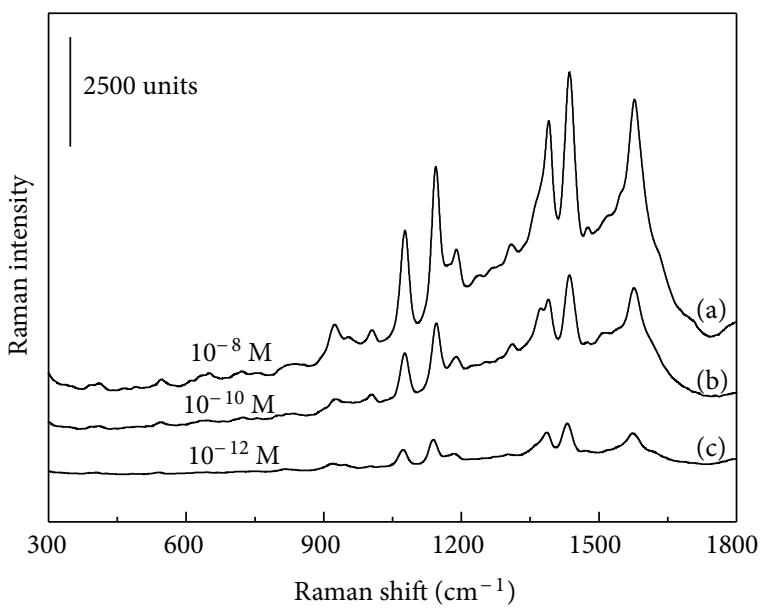

Figure 5: Raman spectra of the 4-ATP on the surface of Ag nanoplates arrays (Figure 2(D)) after immersed different concentration of 4-ATP solution for $30 \mathrm{~min}$, (a) $10^{-8} \mathrm{M}$, (b) $10^{-10} \mathrm{M}$, and (c) $10^{-12} \mathrm{M}$.

is not dropped, which exhibits that the silver nanoplates arrays have high stability using as electrode. In addition, the specific capacitance values can be calculated according to the following equation:

$$
C_{m}=I \times \frac{t}{\Delta V \times m} .
$$

Here, $C_{m}$ is specific capacitance, $I$ is charge/discharge current, $t$ is the discharge time within the voltage difference $(\Delta V)$, and $m$ is the mass of silver nanoplates. From Figure 7 , we can calculate that the specific capacitance is $1270 \mathrm{Fg}^{-1}$. This value is higher compared with other similar electrode [35]. We think that the increase in specific capacitance origins from high touching surface of standing $\mathrm{Ag}$ nanoplates with $\mathrm{KOH}$ solution.

\section{Conclusion}

In summary, we prepared Ag nanoplates on ITO substrate by a seed-decorated electrochemical deposition approach. The density of Ag nanoplates can be controlled through the amount of $\mathrm{Ag}$ seed predecorated. The Ag nanoplates arrays with highest density can be dispersed uniformly on the substrate, which had high roughened surface and displayed an extraordinary superhydrophobicity after a chemical modification. Such Ag nanostructured array shows high SERS activity and hence is a good material for the microdetection device based on SERS effect. In addition, the Ag nanoplates arrays can be used as electrode for electrochemical capacitor with high power density.

\section{Acknowledgments}

The authors acknowledge the financial supports from Natural Science Foundation of China (Grants no. 51101149, 10974203, and 50831005); Anhui Provincial Natural

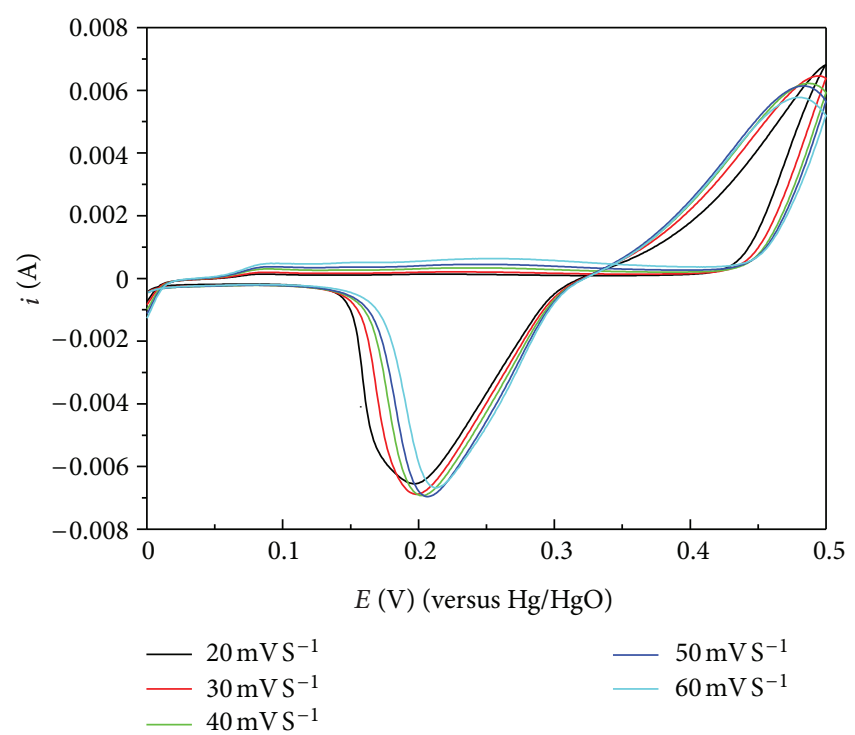

FIGURE 6: The cyclic voltammetry at different scan rate with silver nanoplates arrays (Figure 2(D)) as electrode in $5 \mathrm{M} \mathrm{KOH}$ solution.

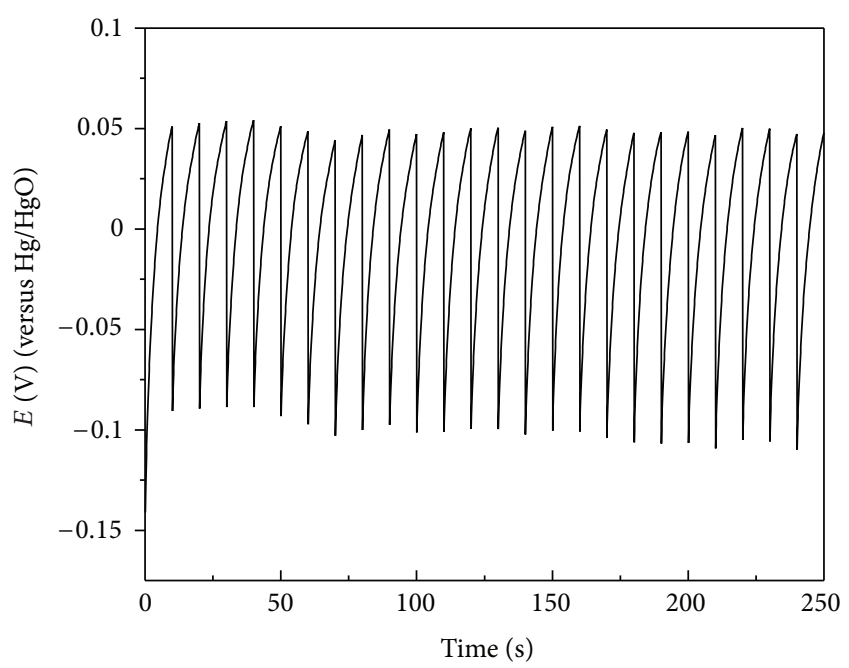

Figure 7: The charge and discharge curve using Ag nanoplates arrays (Figure 2(D)) as electrode. After 25 cycles, the efficiency of charge/discharge is not dropped.

Science Funds for Distinguished Young Scholar (Grant no. 1108085J20), and the National Basic Research Program of China (973 Program, Grant no. 2011CB302103).

\section{References}

[1] W. Fritzsche and T. A. Taton, "Metal nanoparticles as labels for heterogeneous, chip-based DNA detection," Nanotechnology, vol. 14, no. 12, pp. R63-R73, 2003.

[2] N. R. Jana, L. Gearheart, S. O. Obare, and C. J. Murphy, "Anisotropic chemical reactivity of gold spheroids and nanorods," Langmuir, vol. 18, no. 3, pp. 922-927, 2002.

[3] C. Salzemann, I. Lisiecki, A. Brioude, J. Urban, and M. P. Pileni, "Collections of copper nanocrystals characterized by 
different sizes and shapes: optical response of these nanoobjects," The Journal of Physical Chemistry B, vol. 108, no. 35, pp. 13242-13248, 2004.

[4] S. H. Sun, C. B. Murray, D. Weller, L. Folks, and A. Moser, "Monodisperse FePt nanoparticles and ferromagnetic FePt nanocrystal superlattices," Science, vol. 287, no. 5460, pp. 1989-1992, 2000.

[5] N. I. Kovtyukhova and T. E. Mallouk, "Nanowires as building blocks for self-assembling logic and memory circuits," Chemistry, vol. 8, pp. 4354-4363, 2002.

[6] A. Tao, P. Sinsermsuksakul, and P. D. Yang, "Polyhedral silver nanocrystals with distinct scattering signatures," Angewandte Chemie-International Edition, vol. 45, no. 28, pp. 4597-4601, 2006.

[7] S. Habouti, M. Mátéfi-Tempfli, C.-H. Solterbeck, M. Es-Souni, S. Mátéfi-Tempfli, and M. M Es-Souni, "On-substrate, selfstanding Au-nanorod arrays showing morphology controlled properties," Nano Today, vol. 6, no. 1, pp. 12-19, 2011.

[8] F. Xia and L. Jiang, "Bio-inspired, smart, multiscale interfacial materials," Advanced Materials, vol. 20, no. 15, pp. 2482-2858, 2008.

[9] S. K. Yang, W. P. Cai, G. Q. Liu, and H. B. Zeng, "From nanoparticles to nanoplates: preferential oriented connection of Ag colloids during electrophoretic deposition," The Journal of Physical Chemistry C, vol. 113, no. 18, pp. 7692-7696, 2009.

[10] S. K. Yang, W. P. Cai, L. C. Kong, and Y. Lei, "Surface nanometer-scale patterning in realizing large-scale ordered arrays of metallic nanoshells with well-defined structures and controllable properties," Advanced Functional Materials, vol. 20, no. 15, pp. 2527-2533, 2010.

[11] J. F. Li, Y. F. Huang, Y. Ding et al., "Shell-isolated nanoparticleenhanced Raman spectroscopy," Nature, vol. 464, no. 7287, pp. 392-395, 2010.

[12] A. Tao, F. Kim, C. Hess et al., "Langmuir-Blodgett silver nanowire monolayers for molecular sensing using surfaceenhanced Raman spectroscopy," Nano Letters, vol. 3, no. 9, pp. 1229-1233, 2003.

[13] Y. Xiong, J. M. McLellan, J. Chen, Y. Yin, Z. Y. Li, and Y. J. $\mathrm{Xia}$, "Kinetically controlled synthesis of triangular and hexagonal nanoplates of palladium and their SPR/SERS properties," Journal of the American Chemical Society, vol. 127, no. 48, pp. 17118-17127, 2005.

[14] F. Yan, M. B. Wabuyele, G. D. Griffin, A. A. Vass, and T. Vo-Dinh, "Surface-enhanced Raman scattering detection of chemical and biological agent simulants," IEEE Sensors Journal, vol. 5, no. 4, pp. 665-670, 2005.

[15] D. A. Stuart, K. B. Biggs, and R. P. van Duyne, "Surfaceenhanced Raman spectroscopy of half-mustard agent," Analyst, vol. 131, no. 4, pp. 568-572, 2006.

[16] G. T. Duan, W. P. Cai, Y. Y. Luo, Y. Li, and Y. Lei, "Hierarchical surface rough ordered Au particle arrays and their surface enhanced Raman scattering," Applied Physics Letters, vol. 89, no. 18, Article ID 181918, 3 pages, 2006.

[17] G. T. Duan, W. P. Cai, Y. Y. Luo, Y. Li, and Y. Lei, "Electrochemically induced flowerlike gold nanoarchitectures and their strong surface-enhanced Raman scattering effect," Applied Physics Letters, vol. 89, no. 21, Article ID 211905, 3 pages, 2006.

[18] S. M. Nie and S. R. Emory, "Probing single molecules and single nanoparticles by surface-enhanced Raman scattering," Science, vol. 275, no. 5303, pp. 1102-1106, 1997.

[19] J. Jiang, K. Bosnick, M. Maillard, and L. Brus, "Single molecule Raman spectroscopy at the junctions of large Ag nanocrystals,"
The Journal of Physical Chemistry B, vol. 107, no. 37, pp. 9964-9972, 2003.

[20] S. T. Wang, Y. L. Song, and L. Jiang, "Photoresponsive surfaces with controllable wettability," Journal of Photochemistry and Photobiology C, vol. 8, no. 1, pp. 18-29, 2007.

[21] X. Yao, Y. L. Song, and L. Jiang, "Applications of bio-inspired special wettablesurfaces," Advanced Materials, vol. 23, no. 6, pp. 719-734, 2011.

[22] K. S. Liu, X. Yao, and L. Jiang, "Recent developments in bioinspired special wettability," Chemical Society Reviews, vol. 39, pp. 3240-3255, 2010.

[23] N. J. Shirtcliffe, G. McHale, S. Atherton, and M. I. Newton, "An introduction to superhydrophobicity," Advances in Colloid and Interface Science, vol. 161, no. 1-2, pp. 124-138, 2010.

[24] Z. Guo, W. Liu, and B.-L. Su, "Superhydrophobic surfaces: from natural to biomimetic to functional," Journal of Colloid and Interface Science, vol. 353, no. 2, pp. 335-355, 2011.

[25] Y. Li, C. C. Li, S. O. Cho, G. T. Duan, and W. P. Cai, "Silver hierarchical bowl-like array: synthesis, superhydrophobicity, and optical properties," Langmuir, vol. 23, no. 19, pp. 9802-9807, 2007.

[26] Y. Li, W. P. Cai, B. Q. Cao et al., "Two-dimensional hierarchical porous silica film and its tunable superhydrophobicity," Nanotechnology, vol. 17, no. 1, pp. 238-243, 2006.

[27] Y. Li, W. P. Cai, B. Q. Cao et al., "Superhydrophobicity of 2D $\mathrm{ZnO}$ ordered pore arrays formed by solution-dipping template method," Journal of Colloid and Interface Science, vol. 287, no. 2, pp. 634-639, 2005.

[28] V. Gupta and N. Miura, "Electrochemically deposited polyaniline nanowire's network a high-performance electrode material for redox supercapacitor," Electrochemical and Solid-State Letters, vol. 8, no. 12, pp. A630-A632, 2005.

[29] T. L. Sun, L. Feng, X. F. Gao, and L. Jiang, "Bioinspired surfaces with special wettability," Accounts of Chemical Research, vol. 38, no. 8, pp. 644-652, 2005.

[30] X. yao, Q. W. Chen, L. Xu et al., "Bioinspired ribbed nanoneedles with robust superhydrophobicity," Advanced Functional Materials, vol. 20, no. 4, pp. 656-662, 2010.

[31] F. Shi, Y. Y. Song, J. Niu, X. H. Xia, Z. Q. Wang, and X. Zhang, "Facile method to fabricate a large-scale superhydrophobic surface by galvanic cell reaction," Chemistry of Materials, vol. 18, no. 5, pp. 1365-1368, 2006.

[32] F. Shi, Z. Q. Wang, and X. Zhang, "Combining a layer-bylayer assembling technique with electrochemical deposition of gold aggregates to mimic the legs of water striders," Advanced Materials, vol. 17, no. 8, pp. 1005-1009, 2005.

[33] Y. Li, X. J. Huang, S. H. Heo et al., "Superhydrophobic bionic surfaces with hierarchical microsphere/SWCNT composite arrays," Langmuir, vol. 23, no. 4, pp. 2169-2174, 2007.

[34] Y. Li, E. J. Lee, and S. O. Cho, "Superhydrophobic coatings on curved surfaces featuring remarkable supporting force," The Journal of Physical Chemistry C, vol. 111, no. 40, pp. 14813-14817, 2007.

[35] Z. A. Hu, Y. X. Wang, Y. L. Xie, Y. Y. Yang, Z. Y. Zhang, and H. Y. $\mathrm{Wu}$, "Ag nanowires and its application as electrode materials in electrochemical capacitor," Journal of Applied Electrochemistry, vol. 40, no. 2, pp. 341-344, 2010.

[36] G. Liu, W. Cai, L. Kong, G. Duan, and F. Lü, "Vertically crosslinking silver nanoplate arrays with controllable density based on seed-assisted electrochemical growth and their structurally enhanced SERS activity," Journal of Materials Chemistry, vol. 20, no. 4, pp. 767-772, 2010. 
[37] A. B. D. Cassie and S. Baxter, "Wettability of porous surfaces," Transactions of the Faraday Society, vol. 40, pp. 546-551, 1944.

[38] H. H. Wang, C. Y. Liu, S. B. Wu et al., "Highly Raman-enhancing substrates based on silver nanoparticle arrays with tunable sub10 nm gaps," Advanced Materials, vol. 18, pp. 491-495, 2006.

[39] S. J. Lee, A. R. Morrill, and M. Moskovits, "Hot spots in silver nanowire bundles for surface-enhanced Raman spectroscopy," Journal of the American Chemical Society, vol. 128, no. 7, pp. 2200-2201, 2006. 

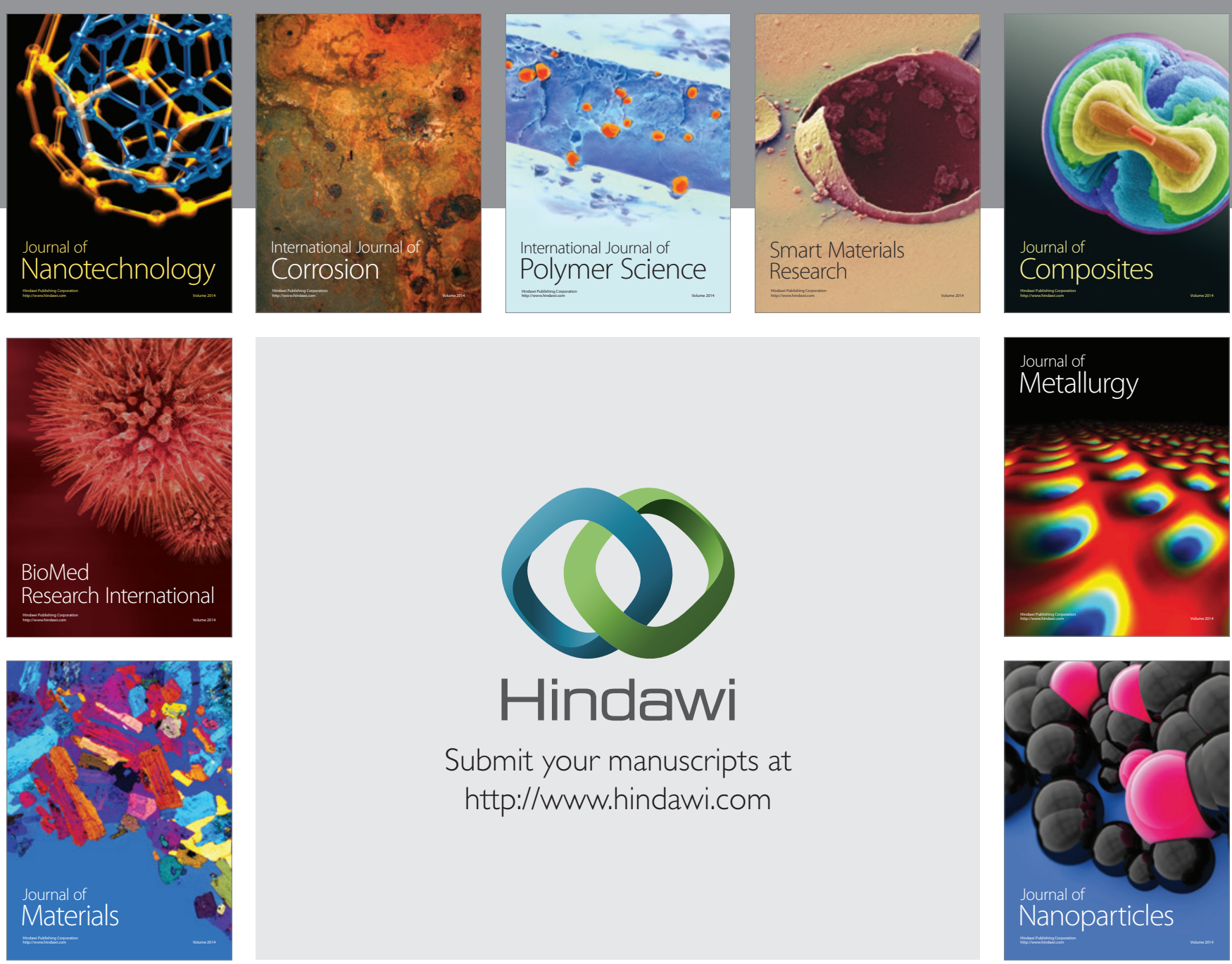

Submit your manuscripts at http://www.hindawi.com
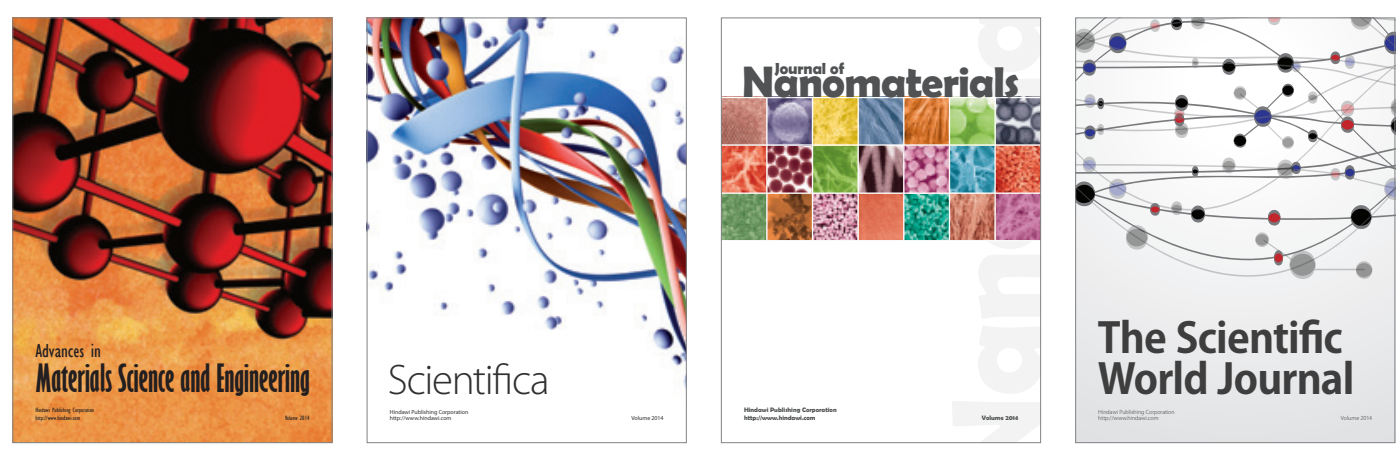

\section{The Scientific World Journal}
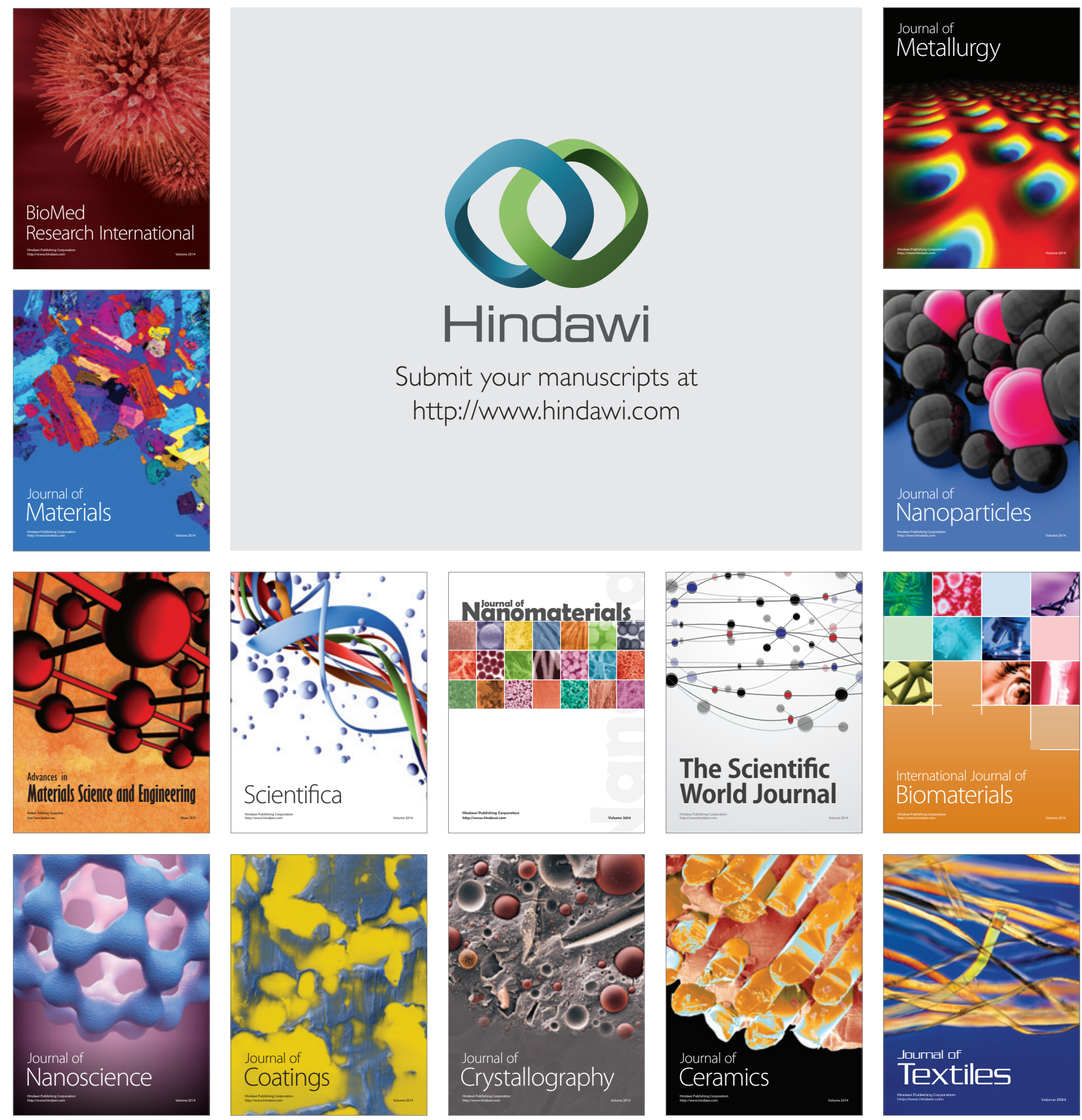